\title{
Clinical Profile and Management in Children with Rheumatic Heart Disease in a Tertiary Cardiac Care Center of Nepal
}

\author{
Poonam Sharma, Urmila Shakya, Sunita K.C., Manish Shrestha
}

Department of Pediatric Cardiology, Shahid Gangalal National Heart Center, Bansbari, Nepal Corresponding Author Poonam Sharma, Department of Pediatric Cardiology, Shahid Gangalal National Heart Center, Bansbari, Kathmandu, Nepal, email: medpoonam@gmail.com

\begin{abstract}
Backgrounds and Aims: Rheumatic heart disease is the commonest acquired heart disease in children of developing world presenting earlier and with a more severe valvular lesion. This study was conducted to assess the clinical and demographic profile, pattern of valvular involvement and management modalities among children with rheumatic heart disease.

Methods: This was a hospital-based observational study, performed at Shahid Gangalal National Heart Center where all children less than 15 years of age with confirmed echocardiographic diagnosis of rheumatic heart disease from June 2015 to May 2016 were evaluated and relevant data were recorded. Results: Two hundred and eleven children were diagnosed with rheumatic heart disease. The mean age was $11.9 \pm 2.2$ years with (100) $47.4 \%$ male and (111) $52.6 \%$ female. The median duration of symptoms among new cases was 1 year (interquartile range of 0.5 to 2 years). The most common symptom was dyspnea present in (187) $88.6 \%$ children followed by palpitation (34.5\%). Mitral valve was most commonly affected in (141) $96.5 \%$ while isolated aortic valve involvement was seen in (8) $3.5 \%$. Isolated mitral regurgitation was the most common lesion present in 118 (55.9\%) children, while $50(23.7 \%)$ children had combination of mitral and aortic regurgitation. Mitral valve was significantly more commonly affected in females and aortic valve commonly affected in males $(\mathrm{p}=0.003)$.

Conclusions: Rheumatic heart disease although declining in developed countries, remains to be the commonest acquired heart disease in developing and underdeveloped countries. Efforts are needed to improve primary and secondary preventive measures.
\end{abstract}

Key Words: Acquired heart disease, children, Rheumatic heart disease.

\section{Introduction}

Congenital heart disease is the commonest cardiac illness in children. ${ }^{1}$ However, acquired heart disease particularly of rheumatic origin remain to have significant issues in developing and underdeveloped countries. The prevalence of Rheumatic heart disease (RHD) in school children varies from 0.7/1000 in Egypt to as high as 24/10000 in the Solomon Islands. ${ }^{2}$ In Nepal few studies done estimates the prevalence of RHD to be 2.5/1000 to 10.2/1000 among school going children., ${ }^{3,4}$

Children are the most vulnerable age group as the lack of awareness among parents and inability to express their symptoms leads to delayed diagnosis causing more severe valvular involvement. In addition lower literacy rate, inadequate family planning, overcrowding and unhealthy hygienic practices in poor countries like Nepal along with inadequate health system often leads to delayed presentation and higher proportion of severe and complicated cases. ${ }^{5}$

Shahid Gangalal National Heart center (SGNHC) is a tertiary cardiac center of Nepal and only one of the two centers in the country that caters to the need of children with cardiac diseases. This study was conducted to assess the clinical and demographic profile, pattern of valvular involvement and management modalities among children presented to SGNHC with RHD.

\section{Methods}

This was a hospital-based prospective observational study, performed at SGNHC, Bansbari, Kathmandu, Nepal from June 2015 to May 2016. All children of less than 15 years of age attending the pediatric cardiac clinic and emergency department with confirmed echocardiographic diagnosis of RHD were included in the study. The diagnosis of RHD was made according to the World Health Federation criteria 2012 for RHD in patients less than 20 years. ${ }^{6}$ Pre-informed consent was taken from the parents/guardians and ethical approval was acquired from the Institutional Review Board of SGNHC. Duplication of cases was avoided by recording the finding of the first presentation during the study period.

Performa was designed to collect patient information including age, gender, address, symptoms and its duration. Treatment received elsewhere before presentation to the hospital and history of established acute rheumatic fever or suggestive symptoms was recorded if present. Details recording of echocardiographic findings, the valve involved along with the severity of the lesion and the modality of treatment (medical versus surgical) was noted.

Data were recorded and analyzed using the IBM SPSS statistics version 20. Continuous variables were expressed as mean and median whereas categorical variables were presented as frequency and compared using chi-square test. 


\section{Results:}

A total of 211 children with RHD attended the pediatric cardiology clinic and emergency department of SGNHC during the study period. Among them 111 children $(52.6 \%)$ were female. The mean age of children with RHD was $11.9 \pm 2.2$ years with the youngest child being 5.9 years and the oldest being 15 years. The mean age of male children was $12.1 \pm 2.0$ years while females were slightly younger with mean age of $11.8 \pm 2.3$ years.
Figure 1 demonstrated the distribution of cases from different districts of Nepal. Children from almost all parts of Nepal have been affected with RHD. However, we did not have any cases from few districts of hilly and mountains of western and far western region of Nepal because of difficulty in access either to the capital or because of lack of awareness regarding the disease in those areas. The maximum number of children was from Rukum followed by Surkhet and Sindhuli.

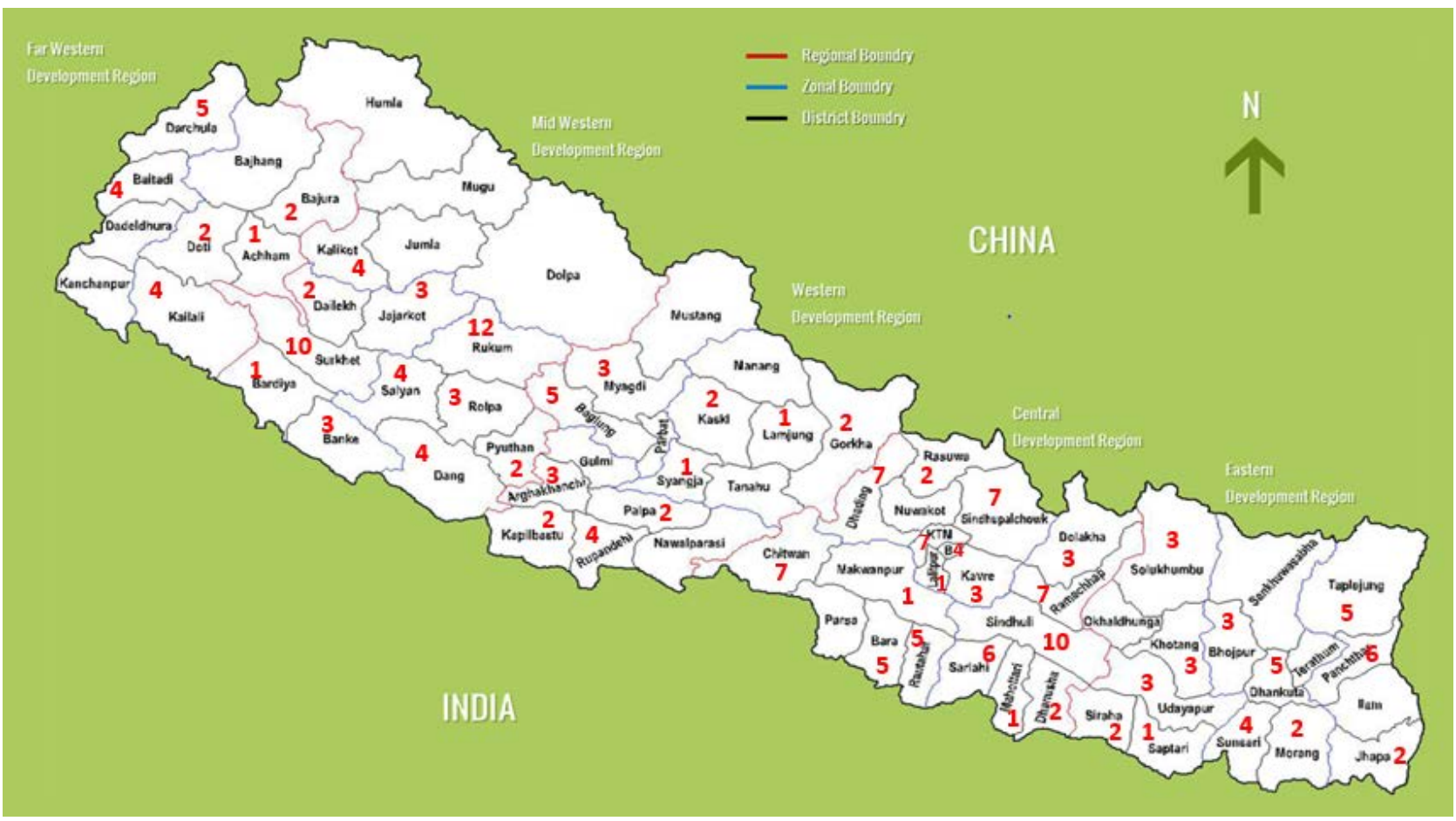

Fig 1: Distribution of children with RHD among various districts of Nepal

Among all cases of RHD, 103 children (48.8\%) presented to the hospital for the first time and $108(51.2 \%)$ children were known cases who come for follow up. The median duration of symptoms among new cases was 1 year (ranging from 1 week to 9 years). Similarly, only 35 children (34\%) who presented to the hospital for the first time received medication from outside while 68 children were referred to this tertiary center without any medication. The most common symptom in children with RHD was dyspnea. Table 1 shows the frequency of various presenting symptoms. Past history suggestive of Rheumatic fever (joint pain and swelling associated with fever) was present only in $47(22.3 \%)$ children. There were a total of 19 children with infective endocarditis during the study period, among whom 16 children (84\%) was those with RHD

Table 1. Frequencies of various symptoms in a child with RHD

\begin{tabular}{|llll|}
\hline Presenting symptoms & $\begin{array}{l}\text { Number } \\
\text { of children }\end{array}$ & Percentage & $\begin{array}{l}\text { Total } \\
\text { (n=935) }\end{array}$ \\
\hline Dyspnea & 187 & 88.6 & 385 \\
\hline Palpitation & 73 & 34.5 & 251 \\
\hline Chronic cough & 54 & 25.5 & 232 \\
\hline Abdominal pain & 38 & 18 & 67 \\
\hline Fever & 24 & 11.3 & \\
\hline Arthritis & 7 & 3.3 & \\
\hline Chorea & 3 & 1.4 & \\
\hline
\end{tabular}

Mitral valve was the most common valve affected with RHD present in $96.5 \%$ of children while isolated aortic valve involvement was seen in eight (3.5\%) children. Table 2 shows the gender distribution of the various valves involved in RHD. Mitral valve involvement was significantly more common in female children whereas aortic valve involvement was significantly more in males $(p=0.003)$. Similarly, Table3depicts the various combinations of valvular involvement in the study population.

\begin{tabular}{|c|c|c|c|c|}
\hline & Male & Female & Total & $\mathrm{p}$ value \\
\hline $\begin{array}{l}\text { Valvular lesion: } \\
\text { - Isolated MV } \\
\text { - Isolated AV } \\
\text { - Combined MV nd AV }\end{array}$ & $\begin{array}{l}59 \\
8 \\
33\end{array}$ & $\begin{array}{l}82 \\
0 \\
29\end{array}$ & $\begin{array}{l}141 \\
8 \\
62\end{array}$ & $\begin{array}{l}0.003 \\
0.003\end{array}$ \\
\hline MR & 85 & 105 & 190 & 0.02 \\
\hline MS & 13 & 18 & 31 & 0.5 \\
\hline $\mathrm{AR}$ & 39 & 29 & 68 & 0.04 \\
\hline AS & 5 & 5 & 10 & 0.86 \\
\hline
\end{tabular}

MV: mitral valve; AV: aortic valve; MR: mitral regurgitation; $M S$ : mitral stenosis; AR: aortic regurgitation; AS: aortic stenosis 
Table 3. Frequencies of different combination of valvular lesions

\begin{tabular}{|lll|}
\hline & Frequency & Percent \\
\hline Isolated MR & 118 & 55.9 \\
\hline Isolated MS & 11 & 5.2 \\
\hline Isolated AR & 7 & 3.3 \\
\hline MR+MS & 12 & 5.7 \\
\hline AR+AS & 1 & 0.4 \\
\hline MR+AR & 50 & 23.7 \\
\hline MR+AS & 1 & 0.5 \\
\hline MS+MR+AR & 3 & 1.4 \\
\hline AR+AS+MR & 3 & 1.4 \\
\hline AR+AS+MS & 2 & .9 \\
\hline MR+MS+AR+AS & 3 & 1.4 \\
\hline Total & 211 & 100 \\
\hline
\end{tabular}

MR: mitral regurgitation; MS: mitral stenosis; AR: aortic regurgitation; AS: aortic stenosis

In this study, $147(69.6 \%)$ children were managed in the outpatient department with oral medications, 24 (11.4\%) children required admission due to either infective endocarditis and/or heart failure and 40 (19\%) were referred to the surgical department for valve repair/replacement.

\section{DISCUSSION}

Rheumatic fever and RHD, though rare in developed nations, is the commonest acquired heart disease in children of poor nations with 470,000 reported new cases and 233000 deaths annually. ${ }^{1}$ As RHD affects the poorest people of the world, they often fail to receive the attention they require. 7 The global burden of RHD varies according to various regions with unreliable estimates due to missing data in many countries and the use of different methods for diagnosis. ${ }^{2}$

Few studies reported, more so only in and around Kathmandu valley to estimate the incidence and prevalence of RHD in Nepalese children. Although the prevalence was initially estimated to be 1 to $1.3 / 1000$ in school children, presence of subclinical carditis in normal children results in much higher prevalence of RHD in the population. ${ }^{4,8,9}$ Shrestha et al in their recent population based study in school going children of Eastern Nepal have reported an alarming prevalence of 10.2/1000 school children. $^{3}$

The mean age of children with RHD was $11.9 \pm 2.2$ years. This is consistent with the result from Nigeria where the mean age of children with RHD was recorded to be $10.8 \pm 3.2$ years. ${ }^{10}$ Females were slightly more affected in this study with male to female ratio of 0.9:1 which is similar to studies done in different parts of the world. ${ }^{11}$ Further we have observed that girls with RHD are slightly younger than boys, similar to observation made by Shrestha et al where RHD was commoner in girls and affecting them at a younger age. ${ }^{3}$

The median duration of symptoms among new cases was 1 year (range, 1 week to 9 years) with one child had symptoms for as long as nine years without seeking any health care facilities. This signifies the delay in health seeking behavior and visiting the health facility only when the disease is severe which drastically increases the morbidity and mortality. ${ }^{12}$ Further only
$22.3 \%$ of children gave history of rheumatic fever. This is similar to children from sub-Saharan countries where Tadele et al have observed that only $25 \%$ of children with RHD ever recall of having a rheumatic fever. ${ }^{13}$

Dyspnea was the most common symptom among children with RHD present in $88 \%$ of children followed by palpitation. This finding is in contrast to that observed by Zhang et al from Uganda where palpitation was the most common observed symptom followed by fatigue and dyspnea. ${ }^{14}$ This could be because their study was done in adult population and children are less likely to notice or verbalize more subjective symptoms like palpitation or fatigue. The symptoms a child presents in the clinic are usually those that are observed by the mother/caretaker. Mitral valve was the most common valve affected with RHD present in $96.5 \%$ of children. Isolated aortic valve involvement was seen in eight $(3.5 \%)$ children. Similarly, mitral valve involvement was significantly more common in female children whereas aortic valvulitis was observed to be more in males. This was similar to the study from eastern Nepal where mitral stenosis was more common in females and aortic regurgitation in males. ${ }^{15}$

Infective endocarditis was observed in $16 \%$ of children with RHD which is the cause for $84 \%$ of cases with infective endocarditis. The association of this deadly and potentially fatal complication with RHD is almost present only in Asian countries. ${ }^{9}$ Studies from India and Turkey have shown RHD to be the most common cause for infective endocarditis representing 33\% to $66 \%$ of $\operatorname{cases}^{16,17,18}$ whereas in Singapore RHD was responsible for only $4 \%$ of endocarditis cases. ${ }^{19}$

RHD is the second commonest cause of open heart surgery in Nepal. ${ }^{20}$ However, valvular surgery in pediatric population has its own difficulties regarding the procedure, the size of the valve and immediate postoperative complications. ${ }^{21}$ Further there is an additional burden of lifelong oral anticoagulants required after valvular replacement therapy along with the complication of valve thrombosis and systemic embolic disasters. ${ }^{22}$ However, 40 children of less than 15 years of age had had to undergo valvular repair/replacement surgery as their symptoms despite adequate and aggressive medical therapy. This is probably because these children presented late in the course of the illness with severely deformed valves and significant dilatation of the heart chambers. This observational study has certain limitation. Being a single center study done it does not reflect the prevalence of the whole country. Further being a tertiary level referral center it may represent only advanced and severe cases that were referred from the periphery. Similarly, we could not analyze the status of pulmonary hypertension and specific chamber dilatation. Finally follow up analysis was not done to look for the outcome after medical/surgical management.

\section{Conclusion}

RHD although in declining phase in developed countries, remains to be the commonest acquired heart disease in poorly developed nations. Its increased incidence is complicated by late presentation to the hospital increasing the severity of valvular involvement of this potentially preventable condition. Proper education and awareness along with effective primary and secondary preventive strategies remain the cornerstone for the control of this common childhood condition.

\section{References:}

1. Chelo D, Nguefack F, Menanga AP, et al. Spectrum of heart diseases in children: an echocardiographic study of 1,666 subjects in a pediatric hospital, Yaounde, Cameroon. Cardiovasc Diagn Ther. 2016;6(1):10-9. 
2. de Dassel JL, Ralph AP, Carapetis JR. Controlling acute rheumatic fever and rheumatic heart disease in developing countries: are we getting closer? Curr Opin Pediatr. 2015;27(1):116-23.

3. Shrestha NR, Karki P, Mahto R, et al. Prevalence of Subclinical Rheumatic Heart Disease in Eastern Nepal. JAMA Cardiol 2016;1(1):89-96.

4. Regmi PR, Wyber R. Prevention of Rheumatic Fever and Heart Disease: Nepalese Experience. Glob Heart. World Heart Federation (Geneva) 2013;8(3):247-52.

5. Feldman T. Rheumatic heart disease. Curr Opin Cardiol. 1996;11(2):126-30.

6. Reményi B, Wilson N, Steer A, et al. World Heart Federation criteria for echocardiographic diagnosis of rheumatic heart. Nat Publ Gr. 2012;9(5):297-309.

7. Maurice J. Rheumatic heart disease back in the limelight. Lancet 2013;382(9898):1085-6.

8. Prajapati D, Sharma D, Regmi PR, et al. Epidemiological Survey of Rheumatic Fever, Rheumatic heart disease and Congenital heart disease among school children in Kathmandu valley of Nepal. Nepalese Heart Journal $2013 ;(1): 1-5$.

9. Carapetis JR. Rheumatic heart disease in Asia. Circulation 2008;118(25):2748-53.

10. Bode-Thomas F, Ige OO, Yilgwan C. Childhood acquired heart diseases in Jos, north central Nigeria. Nigerian Medical Journal. 2013:54(1):51-8.

11. Bhardwaj R, Kandoria A, Marwah R, et al. Prevalence of Rheumatic Fever and Rheumatic Heart Disease in Rural Population of Himachal- A Population Based Study. J Assoc Physicians India. $2012 ; 60: 13-4$.

12. Akinwusi PO, Peter JO, Oyedeji AT, et al. The new face of rheumatic heart disease in South West Nigeria. Int J Gen Med. 2013;6:375-81.

Cite this article as: Poonam Sharma, Urmila Shakya, Sunita K.C, et al. Clinical profile and management of rheumatic heart disease children in a tertiary cardiac care center of Nepal.Nepalese Heart Journal 2016;13(2): 33-36.
13. Tadele H, Mekonnen W, Tefera E. Rheumatic mitral stenosis in children: more accelerated course in subSaharan patients. BMC Cardiovasc Disord. 2013;13:95.

14. Zhang W, Mondo C, Okello E, et al. Presenting features of newly diagnosed rheumatic heart disease patients in Mulago Hospital: a pilot study. Cardiovasc J Afr. 2013;24(2):28-33.

15. Shrestha NR, Pilgrim T, Karki P, et al. Rheumatic heart disease revisited: patterns of valvular involvement from a consecutive cohort in eastern Nepal. J Cardiovasc Med. 2012;13(11):755-9.

16. Agarwal R, Bahl VK, Malaviya AN. Changing spectrum of clinical and laboratory profile of infective endocarditis. J Assoc Physicians India. 1992;40(11):721-3.

17. Garg N, Kandpal B, Garg N, et al. Characteristics of infective endocarditis in a developing country-clinical profile and outcome in 192 Indian patients, 1992-2001. Int J Cardiol 2005;98(2):253-60.

18. Heper G, Yorukoglu Y. Clinical, bacteriologic and echocardiographic evaluation of infective endocarditis in Ankara, Turkey. Angiology.2002;53(2):191-7.

19. Liew WK, Tan TH, Wong KY, et al. Infective endocarditis in childhood: a seven-year experience. Singapore Med J. 2004;45(11):525-9

20. Limbu YR, Maskey A. Current Status of Rheumatic fever and Rheumatic Heart disease in Nepal. J Nepal Med Assoc. 2002;41:514-7.

21. Atik FA, Dias AR, Pomerantzeff PMA, et al. Immediate and long term evolution of valve replacement in children less than 12 years old. Arq Bras Cardiol. 1999;73(5):424-8.

22. Henaine $R$, Roubertie $F$, Vergnat $M$, et al. Valve replacement in children: A challenge for a whole life. Arch Cardiovasc Dis. 2012;105(10):517-28. 